\title{
Caracterización de la población que asiste a casinos en la ciudad de Neiva ${ }^{1}$
}

\author{
José Manuel Agudo Benavides, María Alejandra Martínez Cardoso, Willian Sierra-Barón.
}

Universidad Surcolombiana

\section{Resumen}

En este artículo se identifican las características sociodemográficas de los sujetos que asisten a los casinos en la ciudad de Neiva, Huila. Participaron 313 personas, 223 hombres y 90 mujeres, a los que se les aplicó de forma individual el Cuestionario de juego patológico de South Oaks Gambling Screen (SOGS): Validación Española y el Cuestionario de Variables Psicosociales y Socioeconómicas para personas que juegan en casinos. Los resultados obtenidos en el SOGS sugieren que los participantes pueden llegar a poseer algunos problemas con el juego de azar; en el Cuestionario de Variable Psicosociales y Socioeconómicas gran parte de los participantes son de estado civil soltero, la caracterización sociodemográfica de la población asistente a los casinos de la ciudad de Neiva tiene un mayor predominio de asistencia de ciudadanos de estrato socioeconómico 1 y 2. Finalmente, se encuentra un gran impacto en la vida familiar, económica y laboral del sujeto que asiste a los casinos.

Palabras Claves: Juego, casino, caracterización, neiva-huila

\section{Population Characterization assisting to Casino's Neiva'}

\begin{abstract}
Sociodemographic characteristics were found in people who attend casinos in Neiva's city, Huila. Three hundred and thirteen Neiva citizens, 223 men and 90 women completed two questionnaires: Questionnaire pathological gambling in South Oaks Gambling Screen (SOGS): Spanish Validation and Questionnaire psychosocial variables and socioeconomic for people that play at casinos. The results of questionnaire SOGS suggest that the participants can get to have some problems with gambling; Questionnaire on Psychosocial and Socioeconomic Variable indicate that most of the participants are single marital status, and also, it is a highly prevalent in people who attend to Casinos of socioeconomic one and two. Finally, it has a big impact on family, economic and professional life of people who attends casinos.
\end{abstract}

Keywords: Game, casino, characterization

\footnotetext{
${ }^{1}$ Este artículo es producto del trabajo de grado "Caracterización de la población que asiste a casinos en la ciudad de Neiva", realizada por José Manuel Agudo, Erika Rocio Dueñas, María F. Mosquera y Yafredt León Medina.
} 


\section{Introducción}

La tendencia inherente de las personas en prácticas de socialización se convierte en acciones que claman su interés desde pequeños, debido a que estas prácticas facilitan las relaciones con otros sujetos para el entretenimiento en toda etapa evolutiva de acuerdo con su edad, intereses o afinidades. La recreación mediante el juego es importante en la vida de las personas, sin embargo, los casinos y los juegos de azar son una industria que genera problemáticas a nivel social, ya que es la fuente principal en la incidencia de jugadores patológicos o problemáticas a nivel personal, económica, laboral y familiar en los jugadores que concurren estos establecimientos (Devereux, 1980).

Brieva (2006) y Russel (1984) convergen en que el juego es una actividad que genera placer al ser humano y no se realiza con una finalidad más allá del placer de tal modo que se convierte en una actividad recreativa. Se debe aclarar que el juego no es una adición en tanto que el ser humano aprende a respetar reglas, mejora sus relaciones interpersonales e intrapersonales; el problema se presenta en el instante el cual el apostar prioriza sobre el juego (Brieva, 2006).

En la normatividad colombiana, las modalidades de los juegos de azar y suerte están legalmente constituidos por la Ley 643 del 2001, esta ley establece el régimen propio del monopolio rentístico de juegos de suerte y azar, los cuales corresponden a: Loterías, apuestas permanentes o chance, rifas, juegos promocionales, eventos deportivos, gallísticos, caninos y similares; juegos novedosos, eventos hípicos y juegos localizados. Se conoce como juegos localizados a los lugares que ofertan juegos de azar tales como la ruleta, las maquinas traga monedas, 21 blackjack, entre otros, donde ponen al servicio de la población alternativas de entretenimiento y recreación con la finalidad de generar espacios de diversión $\mathrm{y}$ ocio mediante un intercambio lucrativo.

Para el sujeto que juega apostando de forma frecuente en los casinos, esta actividad se convierte en una fuente de excitación y de ocio que dominará enteramente su vida y que arrastrará una multitud de consecuencias nefastas en diferentes dimensiones del individuo (Custer y Milt, 1986).

El estudio de los juegos de azar ha sido trabajado desde diversas disciplinas tales como la sociología, psicología, antropología, pedagogía y medicina. Los estudios de investigación abordados desde la psicología, arrojan información que relaciona la morbilidad existente entre los casinos y la incidencia de jugadores o la adquisición de problemáticas que interfieren en la calidad de vida de las personas que frecuentan estos establecimientos. La calidad de vida de estas personas está caracterizada por una pérdida del control continua o periódica sobre el apostar, preocupación constante por apostar y obtener dinero para tal fin, un pensamiento irracional en torno al juego y el mantener esta conducta a pesar de las consecuencias adversas que provoque (Roshental \& Lorenz, 1992).

La conducta irracional de desear apostar y de perder el control de sus acciones con ese deseo, afecta por completo al individuo al convertirlo en una persona dependiente a estos juegos, alterando la personalidad e interfiriendo de forma negativa en la economía, familia y vida profesional del jugador (Becoña, Lorenzo, \& Fuente, 1996).

Un estudio de la Federación Andaluza de Jugadores de Azar Rehabilitados (FAJER) identificó que los casinos poseen una facilidad para tentar a los individuos por medio de la suerte y el azar, con atractivos como apuestas riesgosas que generan una mayor emoción en el jugador y crean en el individuo una mínima posibilidad de imaginar que existe riesgo de perder (Salinas y Roa, 2001). Así que quienes empiezan a asistir a casinos con el objetivo de divertirse, pueden llegar adquirir características de adición con el paso del tiempo, ya que estos establecimientos están diseñados para cautivar y mantener a los usuarios, mediante estrategias como aislar el establecimiento del mundo exterior al ofrecer aire acondicionado para mantener una temperatura estable y confortable, aislar el paso de luz natural para que las personas no perciban el cambio de 
horario, brindar de forma gratuita bebidas refrescantes y alcohólicas, además de ofrecer desayuno, almuerzo y cena sin costo alguno (Mercado, 2013).

Pero quizás el mayor atractivo de los casinos es la posibilidad ficticia de enriquecer a las personas al multiplicar los ingresos de forma rápida y fácil, mediante un juego de azar (Garcia, 2008), que poco a poco al realizar las actividades de juego en los casinos genera en el individuo pensamientos erróneos o irracionales que llevan a que el jugador repita la conducta reiteradamente, como lo son la creencia de influir en los resultados, esto a pesar de que los juegos de azar son incontrolables e impredecibles.

El dinero posee un papel importante dentrode los casinos para sus usuarios, al ser la llave de entrada a los diferentes juegos que tientan a los jugados, conlleva que con el fin de financiar los juegos de azar soliciten préstamos con bancos, familiares, amigos o casinos, afectando la economía del individuo o sus allegados, esto ocurre debido a que dejan de lado responsabilidades familiares o personales, solo para seguir destinando fondos a los casinos o a las deudas contraídas en estos con tal de poder seguir apostando, bajo la creencia de que recuperaran lo perdido y además obtendrán grandes ganancias así no tendrán más problemas.

Las repercusiones se encuentran en cualquier ámbito de la vida del jugador; el deterioro en la familia cercana se debe al distanciamiento que se da entre mujer e hijos y el jugador pues, el tiempo que tiene libre le dedica a las actividades de juego trayendo consigo, un aumento del consumo de alcohol y tabaco. De igual manera, la economía familiar se quiebra pues, el jugador adquiere deudas ligadas a la asistencia concurrida en los casinos.

En muchos casos, la pareja del jugador tiene sentimientos de vergüenza y exclusión social debido a que debe de responder por los problemas de juego, las deudas adquiridas por el jugador pueden también ser causa de separación o divorcio. También en ellas se desarrollan sentimientos de ira, depresión, soledad, culpabilidad por el problema de la pareja, confusión, sentimientos de inefectividad como pareja y madre, desesperanza y ruina, e incluso se evidencian casos de suicidio (Lorenz \& Shuttlesworth, 2006).

Por otro lado, los hijos del jugador pueden llegar a ser víctimas de maltrato físico y psicológico, en muchos casos por la frustración del jugador al perder o no poder obtener el suficiente dinero para sostener a su familia. La percepción de los hijos se puede llegar a ser de abandono o faltos de afecto creciendo así en un ambiente de aislamiento, abuso, rechazo, carencia emocional y con dificultades para cubrir las necesidades básicas a raíz de la falta de solvencia económica (Darbyshire, Oster, \& Howard, 2001).

Agregado a lo anterior, aquellos hijos de jugadores, con el fin de establecer un vínculo emocional y afectivo con el pariente distante o por medio de observación (aprendizaje vicario) llegan a participar en actividades de juegos de azar al cumplir la mayoría de edad, pudiendo llegar a convertirse en jugadores patológicos o contraer consecuencias ligadas al juego patológico (Custer \& Milt, 1985).

A nivel laboral se encuentran repercusiones en el individuo que acude a casinos de forma regular, tales como el absentismo laboral, incumplimiento en los horarios laborales, problemas de concentración y disminución del rendimiento, conflictos con los compañeros del trabajo, amenazas de despido o no renovación del contrato, accidentabilidad laboral y robos o estafas en el trabajo. También, es bastante usual que durante las horas laborales los pensamientos de la persona giren en torno al juego, provocando disminución en el rendimiento y productividad, puede darse el caso en que el sujeto al estar inmerso en el juego y las condiciones ambientales que ofrece el casino hacen que pierda el control del tiempo y se sienta incapaz de responder por las obligaciones laborales; otro comportamiento indebido en el ámbito laboral hace referencia a hurtos o estafas por parte del jugador en el trabajo, donde considera el dinero robado un préstamo que será pagado con las ganancias producto de las apuestas. Los conflictos entre compañeros de tra- 
bajo se deben a la irritabilidad del jugador y a las frustraciones en los resultados de juegos de azar, además del deterioro de la relación con los compañeros de trabajo debido a la adquisición de deudas con estos y el no pago de dicha obligación (Fernandez- Montalvo, Baez, y Echeburua, 2000).

Por supuesto, es importante resaltar que cualquier sujeto puede participar en los servicios que ofrecen estos establecimientos y ser beneficiado por las bondades del juego, pero al existir una actividad prolongada y recurrente en estos establecimientos, puede llegar a producir un nivel de interferencia personal en el sujeto, que se reflejan en los aspectos negativos.

Finalmente, el objetivo del artículo consistió en la caracterización de los sujetos que asisten a casinos registrados en la Cámara de Comercio de la ciudad de Neiva durante el primer semestre del año 2015 considerando de gran relevancia la percepción de los jugadores sobre la influencia de las actividades de juego en su economía, trabajo y familia. Al mismo tiempo, se plantea describir los factores sociodemográficos (género, edad, estrato socioeconómico, nivel educativo, estado civil) de los sujetos que asisten a casinos en la ciudad de Neiva. De este modo y con la aplicación de los criterios del South Oaks Gambling Screen se determina una clasificación de los tipos de jugadores de azar que existen en la ciudad de Neiva.

\section{Método}

\section{Participantes}

Participaron de manera voluntaria en la investigación 313 personas de la ciudad de Neiva, Huila, de los cuales 223 (71,5\%) hombres y 90 $(28,75)$ mujeres. Con un rango de edad entre 18 y 78 años.

\section{Instrumentos}

Cuestionario de juego patológico de South Oaks Gambling Screen (SOGS): Validación

\section{Española}

El SOGS es un cuestionario de 20 ítems, el cual sirve para evaluar la dependencia al juego de los participantes. Las opciones de respuesta son dicotómicas, de selección múltiple y/o tipo Likert.

El coeficiente de fiabilidad test-retest obtenido a partir de la validación española en los 472 sujetos fue de 0.98 ( $\mathrm{p}<0.001)$, con un intervalo de 4 semanas. La consistencia interna, obtenida mediante el coeficiente alfa de Cronbach en la muestra total de sujetos es de 0.94, segmentada por los jugadores patológicos $(n=72)$ el alfa era de 0.66 y de los sujetos "normales" de 0.75 .

\section{Cuestionario de variables psicosociales y socioeconómicas para personas que juegan en casinos}

Es un cuestionario de 26 ítems, el cual está dirigido a indagar datos sociodemográficos y factores que pueden ser afectados por las actividades de juegos de azar de forma frecuente en el ámbito laboral, académico, profesional, familiar o económico. Las opciones de respuesta son de selección múltiple, dicotómicas o tipo Likert.

Los instrumentos fueron revisados a través de juicio de expertos.

\section{Resultados}

La caracterización sociodemográfica de la población asistente a los casinos de la ciudad de Neiva refleja el predominio de asistencia de los estratos socioeconómico 1 y $2(79,6 \%)$ (estrato $1=23 \%$; estrato $2=56.5 \%$ ).

En la tabla No.1 se evidencia que la mayor parte de la población participante en el estudio reside en la zona Centro Oriente de la ciudad (comunas 5 y 7) de Neiva, con el $23.6 \%$, seguida de la zona sur (comuna 6) con un $18.2 \%$ y la zona norte (comunas 2 y 9) con un $17.9 \%$, así mismo se encontró que sujetos que residen en lugares periféricos a la ciudad concurren los casinos de 
Neiva y representan el $1.6 \%$ de los participantes del estudio.

Con relación al nivel educativo se observa en la tabla No.2 la población que acude con mayor frecuencia a los casinos en Neiva son personas con un nivel educativo de bachiller completo, con el $27.5 \%$, le sigue el bachillerato incompleto con el $14.1 \%$ y la primaria completa, representada en el $12.5 \%$, además se encuentra que el $11.5 \%$ de los sujetos estudiados poseen un título universitario, por otro lado se evidencia que el
$8.9 \%$ representan sujetos sin formación académica o primaria incompleta

De acuerdo con la tabla No.2 la población asistente con mayor frecuencia a los casinos en Neiva son personas con un nivel educativo de bachiller completo del $41 \%$, posteriormente sigue la educación básica primaria representada en el 18,3\%, además se encuentra que el 19,7\% de los sujetos estudiados están en nivel de educación superior.

El estado civil de las personas que asisten a casinos en Neiva mayoritariamente es soltero

Tabla No. 1.

Ubicación geográfica de los participantes del estudio.

\begin{tabular}{lccc}
\hline Zona de la ciudad & $\begin{array}{c}\text { Comunas que } \\
\text { conforman la } \\
\text { zona }\end{array}$ & Frecuencia & Porcentaje \\
\hline Noroccidente & 1 & 33 & 10.5 \\
Norte & 2 y 9 & 56 & 17.9 \\
Centro & 3 y 4 & 43 & 13.7 \\
Centro oriente & 5 y 7 & 74 & 23.6 \\
Sur & 6 & 57 & 18.2 \\
Sur oriente & 8 & 23 & 7.3 \\
Oriente alto & 10 & 22 & 7.0 \\
Fuera de Neiva & Periferia & 5 & 1.6 \\
Total & & 313 & 100.0 \\
\hline
\end{tabular}

Nota: Los participantes del estudio referían el barrio en donde residían en el cuestionario sociodemográfico, posteriormente en la tabulación de datos se recodificó esta variable según la comuna en donde el barrio perteneciera.

Tabla No. 2.

Nivel educativo alcanzado por los participantes del estudio

\begin{tabular}{lccc}
\hline Titulo alcanzado & Frecuencia & Porcentaje & Porcentaje acumulado \\
\hline Ningún estudio & 10 & 3.2 & 3.2 \\
Primaria & 57 & 18,3 & 21,5 \\
Bachillerato & 130 & 41.6 & 63,1 \\
Técnico o Tecnólogo & 54 & 17,3 & 80,4 \\
Universitario & 62 & 19,7 & 100.0 \\
Total & 313 & 100.0 &
\end{tabular}

Nota: De acuerdo con el título académico alcanzado por los participantes del estudio. Tabla 2. 
$(32,3 \%)$, seguidos por las personas casadas $(28.8 \%)$ y en tercer lugar asisten quienes mantienen una relación conyugal de unión libre $(28,1 \%)$. El 74,4\% de las personas tienen hijos, y sólo el 25,6\% no los tienen. En cuanto a la actividad laboral de los participantes solo el $24,6 \%$ se encuentran desempleados, el restante $(75,4 \%)$ reportaron estar trabajando.

Del total de personas que desempeñan labores formales, el $74.8 \%$ desempeñan labores en entidades privadas, mientras que el $25.2 \%$ en entidades públicas. Además, son más las personas con un contrato indefinido, siendo el $57.1 \%$ de entidades públicas y el $42,3 \%$ de privadas; por otro lado, las personas con contrato por prestación de servicios representan el $25 \%$ en las entidades públicas y un $27 \%$ en la entidad privada.

\section{Resultados de la aplicación del SOGS}

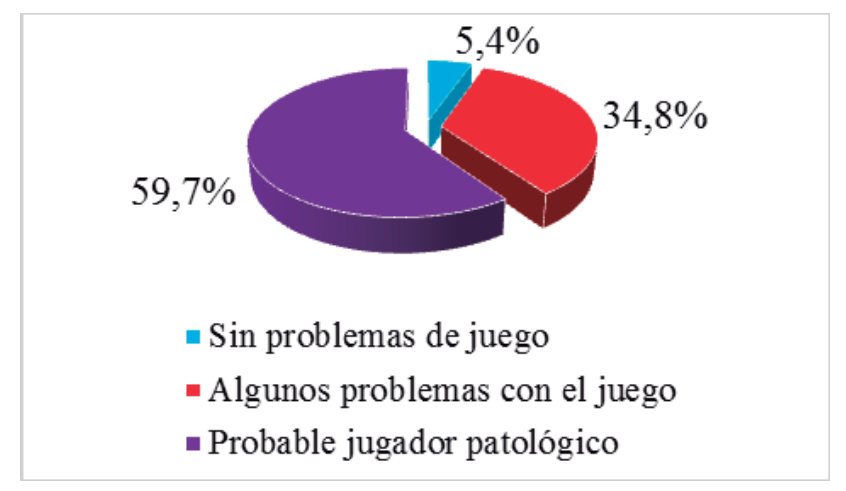

\section{Gráfica 1. Clasificación de sujetos de acuerdo} con los criterios del SOGS

La gráfica No. 1 muestra la clasificación de los tipos de jugadores según el SOGS, donde se encontró que el $59.7 \%$ de los participantes son probables jugadores patológicos, el $34.8 \%$ de ellos poseen algunos problemas con el juego, esto quiere decir que las personas encuestadas probablemente posean problemas con el juego.

\section{Discusión}

El juego como actividad humana y de socialización favorece el adecuado desarrollo de las personas, sin embargo, quienes llegan a participar y frecuentar los juegos de azar ofrecidos por establecimientos con ánimo de lucro (Casinos), pueden llegar a desarrollar una serie de consecuencias que pueden llegar a afectar el bienestar y calidad de vida del participante.

Se identificó que el $74.4 \%$ de los participantes tienen al menos un hijo; con este dato, se infiere que los participantes del estudio son sujetos en su mayoría con responsabilidades familiares, que pueden ser desatendidas o abandonadas por las prácticas de juego del azar, generando conflictos al interior de la familia, como bien lo sugieren Lorenz \& Shuttlesworth, (2006), Lesieur, H. (1993), Custer, R., \& Milt, H. (1985). Robert, M., \& Geis, G. (2006), el estrato socioeconómico hallado $(79,5 \%)$ se puede deber a la adquisición de deudas o la perdida monetaria, esto producto de las practica en juegos de azar, lo cual contrasta con los hallazgos de Cruz et al. (2010), Becoña, (2004), Legarda (1992) y González et al. (2013); pero difiere de lo encontrado por Tallon et al. (2011).

Con relación al nivel de influencia en el ámbito laboral, económico, profesional y personal Tallon et al. (2011) en su investigación determinó que el perfil de jugador usualmente es el de una persona de género masculino, la cual gasta más dinero de la que planeaba a los juegos de azar, sin importar el resultado. Vuelve a jugar y deja perder tiempo de otras actividades a causa del juego. En relación con las problemáticas identificadas, se encontró que el jugador usualmente oculta cuan- do pierde, es criticado por jugar, se siente mal por hacerlo, no quiere jugar, pero lo hace y discute con la familia dichas actividades.

Los resultados obtenidos en la investigación muestran que es necesario indagar a mayor profundidad en la problemática planteada; pues gran parte de los participantes tienen problemas con el juego en algún nivel según los resultados y criterios del cuestionario SOGS, añadiendo esto, que sus diversas responsabilidades profesionales, familiares, económicas y personales se han identificado como afectaciones directas a raíz de las actividades de juego en casino, es decir, los 
jugadores de casinos de Neiva se han percibido afectados por las actividades de juegos de azar en su bienestar social y psicológico.

\section{Conclusiones}

Los resultados sugieren que se presenta en los participantes estudiados una mayor asistencia a los casinos de Neiva en personas de sexo masculino, por sobre el sexo femenino. La edad de los jugadores promedio es de 41,3 años; con relación al estado civil hay un predomino de las personas con algún tipo de relación formal: matrimonio o unión libre, también ligado a esto se encontró que la mayoría de las personas son padres de familia, el estrato sociodemográfico entre los participantes se encuentra entre el estrato 1 y 2 , gran parte de los jugadores tiene un trabajo informal, nivel de educación no mayor a bachillerato.

De acuerdo con los reportes de los sujetos se pudo determinar que las relaciones familiares se han perjudicado notablemente entre los miembros familiares, ya que gran parte de los participantes expresaron en los cuestionarios haber tenido discusiones y problemas con familiares y allegados, esto se da a partir de la frecuencia en la que juegan o la forma como destinan fondos económicos a los juegos de azar.

Por otro lado, el aspecto más afectado en el ámbito profesional es el cumplimiento de responsabilidades, ya que muchos expresaron dejar de lado responsabilidades laborales o académicas para así destinar tiempo a las actividades de juego en casinos. Mientras tanto, en lo económico el aspecto más involucrado fue la adquisición de deudas con el fin financiar el comportamiento de juego en casinos, factor que afectaba la calidad de vida individual e incluso familiar en el jugador. Se encontró además, que las personas que asisten pueden llegar a desarrollar sentimientos de culpa y frustración por los resultados que obtienen en los casinos, sumado a un deseo sin éxito de cesar el comportamiento de juego. Por tanto, el bienestar integral de las personas que juegan se ve afectado por las problemáticas ligadas a los juegos de azar. Haciendo necesario profundizar en un problema identificado, para así crear e implementar planes de contingencia a un problema que, según lo sugerido por los resultados e investigaciones previas a nivel nacional e internacional, podría a futuro constituirse en una problemática mayor.

Finalmente, las tendencias relacionadas al comportamiento de las personas que asisten a los casinos en Neiva vinculadas con el incentivo económico pueden ocasionar conductas que afectan el sujeto en las distintas dimensiones de su vida familiar, laboral, personal y social unidas (Darbyshire, P., Oster, C., \& Howard, C. 2001). Relacionado a esto, Fernandez y Castillo (2004) dan significado a la perdida de los amigos y el ambiente laboral; pues es la familia más cercana al jugador la más perjudicada especialmente, la pareja o hijos del jugador Álvarez et al (2007) afirman que el juego "(...) ha facilitado la aparición de conductas problemáticas que han causado una importante alarma social, lo que se conoce como adicción al juego o juego patológico" (p.43), de igual modo, la búsqueda de acción para su auto deleite (Leiseur, 1993).

\section{Referencias}

Álvarez, A., Perez, M., Salcedo, E., Villahoz, J., \& Fernandéz, R. (2007). Guía clínica: Actuar ante el juego patológico. Sevilla: Junta de Andalucia.

Becoña, E., Lorenzo, M., \& Fuente, J. (1996). Pathological gambling and depression. Psychological reports, 635-640.

Custer, R., \& Milt, H. (1985). Compulsive gambling and the family. New York: Custery H. Milt (Eds).

Custer, R., y Milt, H. (1986). Cuando acabe la suerte: Ayuda para jugadores patológicos y sus familias. Miami: Warner Books Inc.

Darbyshire, P., Oster, C., \& Howard, C. (2001). Children of parent(s) who have a gambling problem: a review of the literature and commentary on research approaches. Health Soc Care Community, 93-185. 
Devereux, E. (1980). Gambling and the social structure: A sociological study of lotteries and horseracing in contemporary America. United States: Ayer Co Pub.

Fernandez, J., y Castillo, A. (2004). Repercusiones familiares del juego patológico: una revisión critica. Salud y Drogas, 149-166.

Fernandez-Montalvo, J., Baez, C., y Echeburua, E. (2000). Ludopatía y trabajo: Análisis de las repercusiones laborales de los ju- gadores patológicos de máquinas traga- perras. Clínica y Salud, 5-14.

Garcia, A. (2008). Ludopatía en México. Revista Mexicana de Psicología, 4547.

Lesieur, H. (1993). Prevalencia, características y tratamiento de los jugadores en los Estados Unidos. Psicología Conductual, 389-407.

Lorenz, V., \& Shuttlesworth, D. (2006). The impact of pathological gambling on the spouse of the gambler. Journal of Community Psichology, 67-76.

Mercado, P. (21 de febrero de 2013). Promociones, activaciones y Below the line.
Obtenido de Informa BTL: http://www. informabtl.com/los-secretos-de-lasve- gas

Robert, M., \& Geis, G. (2006). Criminal behavior and moral issues. Los Angeles: Roxbury Publishing Company.

Rosenthal, R., \& Lorenz, V. (1992). The pathological gambling as criminal offender. Psych, 7-19.

Salinas, J., y Roa, M. (2001). Programa de diagnóstico y evaluación del jugador patológico. Revista internacional de Psicología Clínica y de la Salud, 353370.

South Oaks Gambling Screen. (1994). Cuestionario de juego patológico de South Oaks. Análisis y Modificación de la Conducta, 770-791.

Tallon, J., Angeles, M., Garcia, C., Montero, J., y Perez, M. (2011). Estudiantes universitarios y juego patológico. Un estudio empírico en la Universidad de Murcia. Escritos de Psicología, 50-59. 
